\title{
НЕЛИНЕЙНОТЬ ЗАПИСИ ПРОСТРАНСТВЕННО-ВРЕМЕННОЙ ГОЛОГРАММЫ В ВЫСОКОСЕЛЕКТИВНЫХ ФОТОХРОМНЫХ СРЕДАХ
}

\author{
(Представил П. Саари)
}

В $\left[{ }^{1-5}\right]$ экспериментально и теоретически показано, что в высокоселективных фотохромных средах с фотовыжиганием провалов в неоднородно уширенном спектре примесного поглощения $[6,7]$, возможна голографическая запись как пространственных, так и временных зависимостей светового сигнала и тем самым осуществима 4-мерная пространственно-временная голография ( $r, t$-голография). Достигнуто это благодаря примечательному свойству таких сред запоминать как пространственное, так и спектральное распределение падающего излучения.

В известных средах возможны запись и восстановление временной структуры светового сигнала продолжительностью до $10^{-9}-10^{-8}$ с и с временным разрешением до $10^{-13}$ с. Эти пределы определяются соответственно обратными величинами однородной и неоднородной ширин резонансной линии поглощения примеси. Информационный объем каждого элемента величиной порядка длины волны такой среды определяется, как и в случае спекгральных памятей на основе фотовыжигания провалов, соотношением ширин неоднородной и однородной линий поглощения, которое может достигать $10^{5}-10^{6}$.

Полное теоретическое описание процессов записи и восстановления в $r, t$-голографии требует решения уравнений Максвелла для поля в пространственно и спектрально неоднородной среде, что в общем случае осуществимо только числовыми методами с помощью ЭВМ. Возникает вопрос нахождения приближений, которые давали бы аналитические решения, наиболее близкие к реальной экспериментальной ситуации. $\mathrm{B}\left[{ }^{4}\right]$ развита теория $r, t$-голографии в предположении малого (линейного) изменения оптических свойств пластинки в ходе экспозиции. Соответственная теория качественно хорошо согласуется с экспериментом. Однако в действительности наибольшая эффективность восстановления достигнута в экспериментах, где пропускание образца в ходе записи увеличено в несколько раз $\left[{ }^{2}\right]$. В таком случае предположение о линейном характере записи неприменимо, по крайней мере для всех точек пластинки. Также известно, что при больших дозах монохроматического облучения, когда глубина провала равна $\sim 10 \%$, форма провала искажается, а его ширина увеличивается $\left[{ }^{7}\right]$. Для $r, t$-голографии это означает, что ухудшается точность регистрации спектральных особенностей и уменьшается контрастность голограммной пластинки в спектральном измерении. Таким образом, при записи $r, t$-голограммы нелинейные искажения могут появляться как в пространственных, так и во временных (спектральных) измерениях.

В данной работе развита теория $r, t$-голографии с учетом нелинейного характера процесса записи. При этом в разложении экспонента, определяющего отклик фотохромной среды на дозу облучения, учтены 
члены до второго порядка по времени экспозиции, т. е. учтено наличие нелинейности процесса записи второго порядка. При выводе окончательного результата учтено также наличие конечной ширины неоднородной линии поглощения примеси, т. е. учтена конечная длительность фазовой памяти среды. Следует подчеркнуть, что нелинейность записи, о чем и идет речь, - это нелинейная зависимость пропускания среды от дозы облучения, что известно из теории светочувствительных материалов, а не нелинейное взаимодействие света с веществом, возникающее при применении сильных полей.

\section{1. Распространение световых импульсов в высокоселективной фотохромной среде}

Пусть на твердую матрицу с фотохимически активной примесью падают последовательные лазерные импульсы. Каждую примесную молекулу будем рассматривать как электрический дипольный гармонический осциллятор, который характеризуется эффективной массой $m$, зарядом $e$, коэффициентом затухания (ширина однороднсй линии поглощения) $\Gamma$ и частотой собственных колебаний $\omega_{0}$. Амплитуда колебаний $R\left(\vec{r}, t, \omega_{0}, l\right)$ такого осциллятора во внешнем электрическом поле $l$-го импульса с напряженностью $\varepsilon(\vec{r}, t, l)$ определяется дифференциальным уравнением второго порядка

$\frac{\partial^{2}}{\partial t^{2}} R\left(\vec{r}, t, \omega_{0}, l\right)+2 \Gamma \frac{\partial}{\partial t} R\left(\vec{r}, t, \omega_{0}, l\right)+\omega_{0}^{2} R\left(\vec{r}, t, \omega_{0}, l\right)=\frac{e}{m} \varepsilon(\vec{r}, t, l)$,

где $\vec{r}$ - радиус-вектор данного осциллятора в среде.

При суммировании смещений элементарных дипольных моментов всех осцилляторов в единице объема получается макроскопическая поляризация среды

$$
P(\vec{r}, t, l)=N e \int_{-\infty}^{\infty} g\left(\vec{r}, \omega_{0}, l\right) R\left(\vec{r}, t, \omega_{0}, l\right) d \omega_{0},
$$

где $N$ - концентрация примесных молекул, $\left.g \overrightarrow{(r}, \omega_{0}, l\right)\left.\right|_{l=0}=g_{0}\left(\omega_{0}\right)$ нормированная функция неоднородного распределения осцилляторов по собственным частотам колебаний, предполагаемая независимой от пространственных координат до начала экспозиции. Электрическое поле в среде определяется волновым уравнением Максвелла

$$
\Delta \varepsilon(\vec{r}, t, l)-\frac{1}{c^{2}} \frac{\partial^{2}}{\partial t^{2}} \varepsilon(\vec{r}, t, l)-\frac{4 \pi}{c^{2}} \frac{\partial^{2}}{\partial t^{2}} P(\vec{r}, t, l)=0,
$$

где $\Delta$ - оператор Лапласа, $c$ - скорость света в вакууме*

Предполагаем, что падающие импульсы имеют достаточно слабую интенсивность, но их общее число $l$ является очень большим. Тогда, рассматривая $l$ как непрерывное время экспозиции в соответствии с $\left[{ }^{7}\right]$, записываем уравнение для уменьшения числа фотохимически активных примесных молекул $-d g\left(\vec{r}, \omega_{0}, l\right)$ при возбуждении $d l$ импульсами в виде:

$$
-d g\left(\vec{r}, \omega_{0}, l\right)=g\left(\vec{r}, \omega_{0}, l\right) \times \int_{-\infty}^{\infty} I(\vec{r}, \omega, l) K\left(\omega-\omega_{0}\right) d \omega d l,
$$

\footnotetext{
* Если матрица имеет показатель преломления $n$, то в (3) следует $c$ заменнть на $c / n$.
} 
где $I(\vec{r}, \omega, l)$ - спектральная интенсивность падающего импуль̀а,$\dot{x}-$ коэффициент пропорциональности (с размерностью обратного $I(\vec{r}, \omega, l))$, $K\left(\omega-\omega_{0}\right)$ - форма однородной линии поглощения примесной молекулы, которая при осцилляторной модели (1) имеет вид лоренцовой функции

$$
K\left(\omega-\omega_{0}\right)=\frac{\Gamma}{\pi} \frac{1}{\left(\omega-\omega_{0}\right)^{2}+\Gamma^{2}} .
$$

Свертка с правой стороны (4) учитывает возбуждение примесных молекул светом сложного спектрального состава $I(\vec{r}, \omega, l)$.

Решая (1)-(4) с учетом (5), можно определить электрическое поле $\varepsilon(\vec{r}, t, l)$ в среде в любой момент времени. Для вычисления представим $\varepsilon(\vec{r}, t, l)$ и $R\left(\vec{r}, t, \omega_{0}, l\right)$ в виде интегралов Фурье

$$
\begin{aligned}
\varepsilon(\vec{r}, t, l) & =\frac{1}{2 \pi} \int_{-\infty}^{\infty} \vec{\varepsilon}(\vec{r}, \omega, l) \exp (i \omega t) d \omega, \\
R\left(\vec{r}, t, \omega_{0}, l\right) & =\frac{1}{2 \pi} \int_{-\infty}^{\infty} \bar{R}\left(\vec{r}, \omega, \omega_{0}, l\right) \exp (i \omega t) d \omega,
\end{aligned}
$$

где черточка над символом обозначает Фурье-образ.

Исключая с помощью (2) из выражения (3) макроскопическую поляризацию $P(\vec{r}, t, l)$ и используя (1), (3) и $(6)$, находим уравнение для определения Фурье-компонент электрического поля

$\Delta \vec{\varepsilon}(\vec{r}, \omega, l)+\frac{\omega^{2}}{c^{2}} \vec{\varepsilon}(\vec{r}, \omega, l)+\frac{4 \pi \omega^{2} e^{2} N}{c^{2} m} \int_{-\infty}^{\infty} \frac{g\left(\vec{r}, \omega_{0}, l\right) d \omega_{0}}{\omega_{0}^{2}-\omega^{2}+2 i \Gamma \omega} \vec{\varepsilon}(\vec{r}, \omega, l)=0$.

Для решения (7) представим $\vec{\varepsilon}(\vec{r}, \omega, l)$ в виде

$$
\vec{\varepsilon}(\vec{r}, \omega, l)=\overrightarrow{\varepsilon^{\prime}}(\vec{r}, \omega, l) \exp (-\overrightarrow{i k} \vec{r}),
$$

где $\overrightarrow{\varepsilon^{\prime}}(\vec{r}, \omega, l)-$ медленно меняющаяся амплитуда, $\exp (-\overrightarrow{i k} \vec{r})-$ быстро осциллирующая фаза и $\vec{k}-$ волновой вектор света в вакууме: $|\vec{k}|=\frac{\omega}{c}$. Пусть световой импульс, распространяющийся в направлении оси $z$, падает на фотохромную среду в плоскости с координатой $z=0$. Тогда, заменяя в (7) $\Delta$ на $\frac{\partial^{2}}{\partial z^{2}}$ и в соответствии с приближением медленно изменяющихся амплитуд, пренебрегая вторичным производным амплитуды $\overrightarrow{\varepsilon^{\prime}}(\vec{r}, \omega, l)$, получим дифференциальное уравнение первого порядка

$$
\frac{\partial}{\partial z} \overrightarrow{\varepsilon^{\prime}}(\vec{r}, \omega, l)=-\frac{i \pi e^{2} N}{c m} \int_{-\infty}^{\infty} \frac{g\left(\vec{r}, \omega_{0}, l\right) d \omega_{0}}{\omega_{0}-\omega+i \Gamma} \vec{\varepsilon}^{\prime}(\vec{r}, \omega, l),
$$

где учтено, что в случае резонанса $\omega_{0}^{2}-\omega^{2} \approx 2 \omega\left(\omega_{0}-\omega\right)$. 
Оิсуществляя интегрирование в (4) и (9), найдем для определенйя функции неоднородного распределения осцилляторов и амплитуды светового поля в среде систему интегральных уравнений

$$
\begin{gathered}
g\left(\vec{r}, \omega_{0}, l\right)=g_{0}\left(\omega_{0}\right) \exp \left[-x \int_{-\infty}^{\infty} \int_{0}^{l} I(\vec{r}, \omega, l) K\left(\omega-\omega_{0}\right) d l d \omega\right] \\
\overrightarrow{\varepsilon^{\prime}}(\vec{r}, \omega, l)=\overrightarrow{\varepsilon_{0}^{\prime}}(x, y, \omega) \exp \left[-\frac{i \pi e^{2} N}{c m} \int_{-\infty}^{\infty} \frac{\int_{0}^{z} g\left(\vec{r}, \omega_{0}, l\right) d z d \omega_{0}}{\omega_{0}-\omega+i \Gamma}\right],
\end{gathered}
$$

где $I \vec{r}, \omega, l)=\left|\vec{\varepsilon}^{\prime}(\vec{r}, \omega, l)\right|^{2}$. Здесь $\bar{\varepsilon}_{0}^{\prime}(x, y, \omega)=\left.\bar{\varepsilon}^{\prime}(\vec{r}, \omega, l)\right|_{z=0}-$ амплитуда светового поля до пластинки, предполагаемая одинаковой для всех импульсов.

При описании голографического процесса в рассматриваемой среде нам необходимо решить систему (10) два раза (при описании процесса записи и восстановления соответственно). В общем случае это сделать невозможно, поскольку система (10) не имеет точного аналитического решения. Подходящими оказываются следующие приближения.

1. При описании выжигания голограммы не будем учитывать ослабления амплитуды падающего света в среде и дифракции света в ходе выжигания, т. е. пренебрегаем вторым уравнением системы (10).

2. При считывании голограммы не будем учитывать выжигание примесных центров считывающим импульсом, т. е. пренебрегаем первым уравнением в (10).

Применение таких приближений допустимо, если считывание голограммы по сравнению с записью производится на слабых экспозициях. В реальной экспериментальной ситуации последнее требование должно быть выполнено, поскольку в противном случае сильные считывающие импульсы «вымоют» записанную в среду информацию. Тогда получаем от системы (10) два самостоятельных уравнения

$$
g\left(x, y, \omega_{0}, l\right)=g_{0}\left(\omega_{0}\right) \exp \left[-x l \int_{-\infty}^{\infty} I(x, y, \omega) K\left(\omega-\omega_{0}\right) d \omega\right]
$$

и

$$
\bar{\varepsilon}^{\prime}(\vec{r}, \omega)=\bar{\varepsilon}_{0}^{\prime}(x, y, \omega) \exp \left[-\frac{i \pi e^{2} N}{m c} \int_{-\infty}^{\infty} \frac{z g\left(x, y, \omega_{0}, l\right) d \omega_{0}}{\omega_{0}-\omega+i \Gamma}\right],
$$

первое из которых определяет распределение поглощающих центров в голсграммной пластинке после окончания процесса записи (с $l$ импульсами спектральной интенсивности $I(x, y, \omega))$, а второе описывает считывание такой пластинки (с импульсом спектральной характеристики

$\left.\bar{\varepsilon}_{0}^{\prime}(x, y, \omega)\right)$. Из (11) видно, что запись информации в рассматриваемой среде осуществляется с помощью модуляции функции неоднородного распределения осцилляторов.

Следует отметить, что представленная здесь теория действительна с незначительными уточнениями и в случае записи с помощью одной или нескольких достаточно сильных световых импульсов. Например, удаляя от (10) зависимость по $l$, т. е. полагая $l=1$ и $I(\vec{r}, \omega, l)=I(\vec{r}, \omega)$, получаем систему, описывающую запись с одним сильным импульсом. В случае нескольких импульсов полученную систему необходимо поочередно решать отдельно для каждого проходящего импульса. 


\section{2. Учет нелинейности в описании процессов записи пространственно-временных голограмм}

Пусть элемент фотохромной среды в виде пластинки толщиной $d$ находится между плоскостями $z=0$ и $z=d$. Запись $r, t$-голограмм осуществляем с помощью двух световых полей: опорного и предметного (сигнального) импульсов, которые направляем на пластинку так, чтобы их волновые векторы составляли с осью $z$ равные, но противоположного знака малые углы $\theta$ и - $\theta$ (см. рис. 1). Временную шкалу определяем так, что в момент $t=0$ передний фронт предметного импульса $S(\vec{r}, t)$ коснется пластинки. Опорный импульс $R \overrightarrow{(r}, t)$ выбираем $\delta$-образной формы с плоским волновым фронтом, достигающим точки $\vec{r}=0$ в момент $t=t_{R}$. Суммарная амплитуда света, падающего на пластинку, равна

$E(\vec{r}, t)=S(\vec{r}, t)+R(\vec{r}, t)=S\left(x, y, t-\frac{\vec{n}_{S} \vec{r}}{c}\right)+R_{0} \delta\left(t-t_{R}-\frac{\vec{n}_{R} \vec{r}}{c}\right)$,

где $\vec{n}_{\mathrm{S}}=(-\sin \theta, 0, \cos \theta)$ и $\vec{n}_{R}=(\sin \theta, 0, \cos \theta) \quad$ - единичные векторы в направлении распространения сигнального и опорного импульсов, $R_{0}$ - амплитуда опорного импульса.

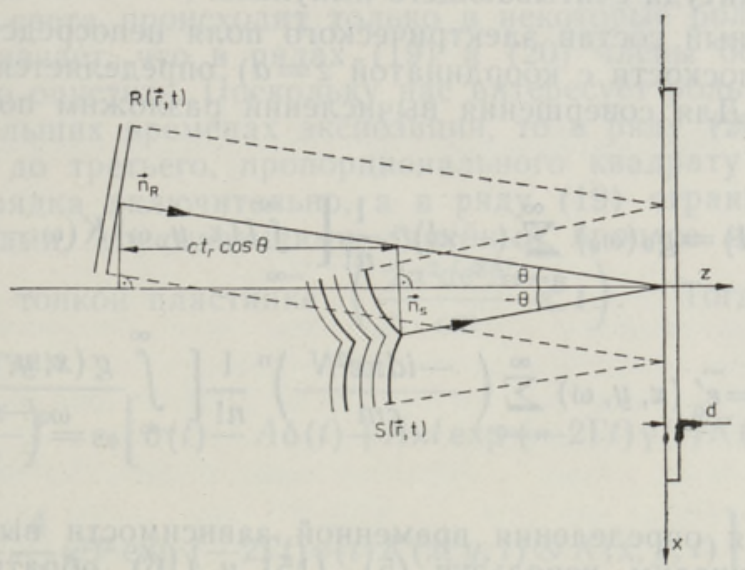

Рис. 1. Запись пространственно-временной голограммы. Спектральная голограмма выжигается с помощью предметного импульса $S(\vec{r}, t)$ и $\delta$-образного опорного импульса $R(\vec{r}, t)$, падающего на пластинку с задержкой $t_{R} \cdot \vec{n}_{s}$ и $\vec{n}_{R}-$ единичные векторы в направлении распространения соответствующих импульсов, $d-$ толщина пластинки.

Запись и считывание голограммы будем рассматривать в приближении тонкой пластинки. Это означает, что либо толщина пластинки $d$, либо угловое расстояние между волновыми векторами сигнального и опорного импульсов должны быть достаточно малы: $(2 \sin \theta)^{2} d \leqslant \frac{2 \pi c}{\omega_{m}}$, где $\omega_{m}$ - максимальная частота света, падающего на голограмму. Таким образом, считаем, что действительны следующие приближенные выражения:

$$
\sin \theta \approx \theta \quad \text { и } \quad \cos \theta \approx 1 .
$$


Следовательно, $\vec{n}_{\mathrm{S}} \approx(-\hat{\theta}, 0,1), \vec{n}_{R} \approx(\theta, 0,1)$.

В соответствии с (11) в голограммной пластинке фиксируется непосредственно спектральная интенсивность $I(x, y, \omega)$ падающего света. Используя (14), из (13) находим:

$$
I(x, y, \omega)=I_{S}(x, y, \omega)+R_{0}^{2}+
$$

$+R_{0}\left[\bar{S}^{*}(x, y, \omega) \exp \left(-i \omega\left(t_{R}+\frac{2 \theta x}{c}\right)\right)+\bar{S}(x, y, \omega) \exp \left(i \omega\left(t_{R}+\frac{2 \theta x}{c}\right)\right)\right]$,

где $I_{S}(x, y, \omega)$ - спектральная интенсивность предметного импульса. -Звездочка означает комплексно-сопряженную величину.

Переходим теперь к рассмотрению процесса восстановления записанного сигнала. Пусть в направлении опорного импульса $\vec{n}_{R}$ на пластинку падает считывающий импульс, который имеет спектральную характеристику, идентичную с опорным

$$
\overline{\varepsilon_{0}^{\prime}}(x, y, \omega)=\varepsilon_{0} \exp \left(-\frac{i \theta x \omega}{c}\right)
$$

где $\varepsilon_{0}$ - амплитуда считывающего импульса.

Спектральный состав электрического поля непосредственно за пластинкой (в плоскости с координатой $z=d$ ) определяется выражениями (11) и (12). Для совершения вычислений разложим последние в степенные ряды

$$
g\left(x, y, \omega_{0}, l\right)=g_{0}\left(\omega_{0}\right) \sum_{n=0}^{\infty}(-x l)^{n} \frac{1}{n !}\left[\int_{-\infty}^{\infty} I(x, y, \omega) K\left(\omega-\omega_{0}\right) d \omega\right]^{n}
$$

и

$$
\left.\bar{\varepsilon}^{\prime}(\vec{r}, \omega)\right|_{z=d}=\bar{\varepsilon}_{0}^{\prime}(x, y, \omega) \sum_{n=0}^{\infty}\left(\frac{-i d \pi e^{2} N}{c m}\right)^{n} \frac{1}{n !}\left[\int_{-\infty}^{\infty} \frac{g\left(x, y, \omega_{0}, l\right) d \omega_{0}}{\omega_{0}-\omega+i \Gamma}\right]^{n} .
$$

Теперь для определения временной зависимости выходного светового поля вычислим, используя (5), (15) и (16), обратное Фурье-преобразование рядов (17) и (18)

$$
\begin{gathered}
\varepsilon^{\prime}\left(\vec{r}, t+\frac{\theta x}{c}\right)= \\
=\varepsilon_{0}\left\{\delta(t)+\sum_{n=1}^{\infty}\left(\frac{-2 \pi^{2} d e^{2} N}{c m}\right)^{n} \frac{1}{n !}[\gamma(t) \exp (-\Gamma t) g(x, y, t, l)]^{n \otimes}\right\},
\end{gathered}
$$

где

$$
g(x, y, t, l)=g_{0}\left\{\delta(t)+\sum_{n=1}^{\infty} \frac{(-x l)^{n}}{n !}[\exp (-\Gamma t) K(x, y, t)]^{n \otimes}\right\} .
$$

Здесь индексом $n \otimes$ обозначена автосвертка $n$-го порядка, стоящего в скобках выражения, $\delta(t)$ - дельта-функция Дирака, $\gamma(t)$ - ступенчатая функция Хэвисайда и $K(x, y, t)-$ функция автокорреляции падающего света (13) 


$$
\begin{gathered}
K(x, y, t)=K_{S}(x, y, t)+R_{0}^{2} \delta(t)+ \\
+R_{0} S^{*}\left(x, y,-t+t_{R}+\frac{2 \theta x}{c}\right)+R_{0} S\left(x, y, t+t_{R}+\frac{2 \theta x}{c}\right),
\end{gathered}
$$

где $K_{\mathrm{S}}(x, y, t)=\int_{-\infty}^{\infty} S^{*}(x, y, \tau) S(x, y, t+\tau) d \tau \quad$ - функция автокорреляции предметного импульса. При получении (19) и (20) использованы формулы

$$
\begin{gathered}
\int_{-\infty}^{\infty} \frac{\exp (i \omega t) d \omega}{\omega_{0}-\omega+i \Gamma}=-2 \pi i \gamma(t) \exp (-\Gamma t) \exp \left(i \omega_{0} t\right), \\
\frac{\Gamma}{\pi} \int_{-\infty}^{\infty} \frac{\exp \left(i \omega_{0} t\right) d \omega_{0}}{\left(\omega_{0}-\omega\right)^{2}+\Gamma^{2}}=\exp (-\Gamma|t|) \exp (i \omega t),
\end{gathered}
$$

а также предположено, что функция неоднородного распределения осцилляторов по собственным частотам колебаний до начала экспозиции $g_{0}\left(\omega_{0}\right)$ является постоянной в спектральной области предметного импульса: $g_{0}\left(\omega_{0}\right)=g_{0}$.

Запись выходного сигнала в виде (19) учитывает дифракцию света произвольно высокого порядка. В действительности обычно значительное рассеяние света происходит только в некоторые более низкие. порядки. Это означает, что в рядах (19) и (20) члены более высокого порядка можно опустить. Поскольку нас интересует роль нелинейности записи при больших временах экспозиции, то в ряду (20) будем учитывать члены до третьего, пропорционального квадрату времени экспозиции $l$, порядка включительно, а в ряду (19) ограничимся двумя первыми членами, описывающими линейный процесс восстановления при оптически тонкой пластинке $\left(\frac{2 \pi^{2} d e^{2} N}{\mathrm{~cm}} \ll 1\right)$. Тогда для выходного поля получим

$$
\begin{gathered}
\varepsilon^{\prime}\left(\vec{r}, t+\frac{\theta x}{c}\right)=\varepsilon_{0}[\delta(t)-A \delta(t)+A x l \exp (-2 \Gamma t) \gamma(t) K(x, y, t)- \\
\left.-\frac{A}{2} x^{2} l^{2} \exp (-2 \Gamma t) \gamma(t) K(x, y, t) \otimes K(x, y, t)\right],
\end{gathered}
$$

где $\otimes$ означает свертку, $A=\frac{2 \pi^{2} d e^{2} N g_{0}}{\mathrm{~cm}}$ и из-под знака свертки выведено медленное затухание $\exp (-\Gamma t)$.

В сумме (23) особый интерес представляют третий и четвертый слагаемые, которые содержат информацию (через функции корреляции) о записанном сигнале. Последний из них описывает влияние нелинейности записи при считывании голограммы.

\section{3. Проявление нелинейности записи при восстановлении пространственно-временной голограммы}

При определении восстановленного светового поля используем формулу (23). Учитывая (21), находим, что сумма (23) состоит из 22 членов. Сгруппируем эти члены по углам отклонения волновых векторов от направления распространения опорного импульса (см. рис. 2).

1. Прямо, без отклонения, проходит пластинку часть считывающего импульса, описываемой суммой 


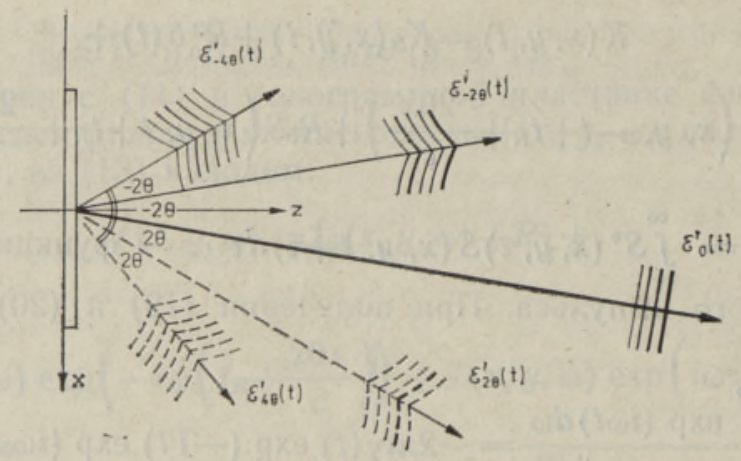

Рис. 2. Восстановление пространственно-временной голограммы. При считывании голограммы, записанной с опережающим опорным импульсом $\left(t_{R}<0\right)$, кроме распространяющегося прямо и без задержки импульса $\varepsilon_{0}^{\prime}(t)$, в моменты времени $\left|t_{R}\right|$ и $\left|2 t_{R}\right|$ возникают рассеянные соответственно под углами $-2 \theta$ и $-4 \theta$ восстановленные сигнальные импульсы $\varepsilon^{\prime}-2 \theta(t)$ и $\varepsilon_{-4 \theta}^{\prime}(t)$. Штрихами изображены дифракционные порядки, возникающие при считывании голограммы, записанной запоздавшим опорным импульсом $\left(t_{R}>0\right)$. При этом восстановленные световые сигналы формируются как с обращенными волновыми фронтами, так и с обращенным ходом времени. Соответственно интенсивностям разные порядки дифракции обозначены линиями разной толщины.

$$
\begin{gathered}
\varepsilon_{0}^{\prime}\left(\vec{r}, t+\frac{\theta x}{c}\right)=\varepsilon_{0} \delta(t)[1-A]+\varepsilon_{0} A x l y(t) \exp (-2 \Gamma t) \times \\
\times\left\{R_{0}^{2} \delta(t)+K_{S}(\vec{r}, t)-\frac{x l}{2}\left[R_{0}^{4} \delta(t)+2 R^{2} K_{S}(x, y, t)+\right.\right. \\
\left.\left.+K_{S}(x, y, t) \otimes K_{S}(x, y, t)\right]\right\} .
\end{gathered}
$$

Распространяющееся прямо световое поле возникает непосредственно после прохождения пластинки считывающим импульсом и не зависит от знака и величины задержки $t_{R}$. Первое слагаемое в (24) описывает ослабление амплитуды считывающего импульса в неэкспонированнсй пластинке, второе - просветление пластинки из-за выжигания части поглощающих центров. Из второго слагаемого видно, что в связи с нелинейностью процесса выжигания прозрачность пластинки не возрастает равномерно с увеличением экспозиции. По своему характеру световое поле (24) является ослабленным считывающим импульсом с растянутым по корреляционным функциям предметного импульса хвостом.

2. Структура светового сигнала, дифрагированного под углом $-2 \theta$, определяется суммой

$$
\begin{gathered}
\varepsilon_{-2 \theta}^{\prime}\left(x, y, t+\frac{\theta x}{c}\right)=\varepsilon_{0} A l x \gamma(t) \exp (-2 \Gamma t) R_{0} S\left(x, y, t+t_{R}+\frac{2 \theta x}{c}\right)- \\
-\varepsilon_{0} A \frac{x^{2} l^{2}}{\rho} \gamma(t) \exp (-2 \Gamma t)\left[S\left(x, y, t+t_{R}+\frac{2 \theta x}{c}\right) R_{0}^{3}+R_{0} K_{S}(x, y, t) \otimes\right. \\
\left.\otimes S\left(x, y, t+t_{R}+\frac{2 \theta x}{c}\right)\right] .
\end{gathered}
$$

Световое поле (25) распространяется в направлении предметного импульса. Первый член в сумме (25) описывает восстановленный сигналь- 
ный импульс в прредположении линейного процесса записи, а второй учитывает ослабление и искажение импульса из-за нелинейности. Видно, что сигнал (25) рассеивается под углом - $2 \theta$ в момент времени $\left|t_{R}\right|$. Из-за обрезающего действия функции $\gamma(t)$ это происходит только в случае если $t_{R}<0$, т. е. когда опорный импульс-подается на пластинку до сигнального. Из (25) следует также возрастающая роль второго (нелинейного) члена с увеличением числа экспонирующих импульcoB.

3. Дифракцию под углом $2 \theta$ описывает формула

$$
\begin{gathered}
\varepsilon_{2 \theta}^{\prime}\left(x, y, t+\frac{\theta x}{c}\right)=\varepsilon_{0} A x l_{\gamma}(t) \exp (-2 \Gamma t) R_{0} S^{*}\left(x, y,-t+t_{R}+\frac{2 \theta x}{c}\right)- \\
-\varepsilon_{0} A \frac{x^{2} l^{2}}{2} \gamma(t) \exp (-2 \Gamma t)\left[S^{*}\left(x, y,-t+t_{R}+\frac{2 \theta x}{c}\right) R_{0}^{3}+R_{0} K_{S}(x, y, t) \otimes\right. \\
\left.\otimes S^{*}\left(x, y,-t+t_{R}+\frac{2 \theta x}{c}\right)\right] .
\end{gathered}
$$

Сигнал (26) появляется только тогда, когда запись голограмм проведена с запоздавшим опорным импульсом $\left(t_{R}>0\right)$. Он отличается от сигнала (25) обращенным волновым фронтом и тем, что соответствующие ему события разыгрываются в обратной временной последовательности.

4. Дифрагированный под углом $-4 \theta$ импульс определяется выражением:

$$
\varepsilon_{-4 \theta}^{\prime}\left(x, y, t+\frac{\theta x}{c}\right)=\varepsilon_{0} A \frac{x^{2} l^{2}}{2} \gamma(t) \exp (-2 \Gamma t) R_{0}^{2} K_{1}\left(x, y, t+2 t_{R}+\frac{4 \theta x}{c}\right),
$$

где $K_{1}(x, y, t)=\int_{-\infty}^{\infty} S(x, y, \tau) S(x, y, t-\tau) d \tau-$ автосвертка предметного импульса,

Сигнал (27) формируется с задержкой $\left|2 t_{R}\right|$ по отношению считывающего импульса и только при выполнении условия $t_{\bar{R}}<0$.

5. Под углом $4 \theta$ дифрагирует импульс

$$
\varepsilon_{4 \theta}^{\prime}\left(x, y, t+\frac{\theta x}{c}\right)=\varepsilon_{0} A \frac{x^{2} l^{2}}{2} \gamma(t) \exp (-2 \Gamma t) R_{0}^{2} K_{1}^{*}\left(x, y,-t+2 t_{R}+\frac{4 \theta x}{c}\right) \text {. }
$$

Рассеивание под углом $4 \theta$ возникает с задержкой $2 t_{R}$ при условии $t_{R}>0$.

Формулы (27) и (28) описывают световые импульсы второго порядка дифракции. Причиной их возникновения является нелинейность процесса записи. Хорошо известно, что в традиционной голографии это ведет к появлению дополнительных световых пучков в восстановленном поле, распространяющихся под углами $\pm 4 \theta$ по отношению к направлению падения считывающей волны. Из $(27)$ и $(28)$ видно, что аналогичный эффект имеет место и в $r, t$-голографии, но с тем отличием, что здесь в направлениях $\pm 4 \theta$ формируется не записанный сигнал, а его автосвертка по времени, имеющая задержку $\left|2 t_{R}\right|$.

Наличие коэффициента $\exp (-2 \Gamma t)$ в формулах $(25)-(26)$ учитывает конечную продолжительность времени фазовой релаксации среды (обратная величина коэффициента затухания осциллятора Г). Этим максимальная допустимая длительность записываемого сигнала (общая продолжительность одной пары предметного и опорного импульсов) яв- 
ляется ограниченной. Видно, что уменьшение по времени амплитуды восстановленного сигнала происходит со скоростью $2 \Gamma$, в два раза превышающей скорость фазовой релаксации среды. Это объясняется тем, что для получения восстановленного сигнала необходимо действовать на фотохромную среду световым импульсом два раза (при записи и при считывании). Учет релаксации в обоих случаях и ведет к двойной скорости затухания восстановленной волны. На спектральном языке этому соответствует хорошо известная удвоенная ширина провала $\Delta=2 \Gamma$ при монохроматическом выжигании спектральных провалов $\left[{ }^{7-9}\right]$. Таким образом, восстановленные импульсы, возникающие с задержкой $\left|t_{R}\right|$ по отношению к считывающим, в момент своего образования по амплитуде в $\exp \left(-2 \Gamma t_{R}\right)$ раз, а по интенсивности соответственно в $\exp \left(-4 \Gamma t_{R}\right)$ раз слабее. Поскольку импульс, распространяющийся под углом $4 \theta$, имеет задержку $\left|2 t_{R}\right|$, то он ослаблен по интенсивности в $\exp \left(-8 \Gamma t_{R}\right)$ раз. Следовательно, для экспериментального обнаружения такого сигнала временная задержка $\left|t_{R}\right|$ должна быть по возможности короткой.

Более точное определение выходного поля требует в суммах (19) и (20) учета членов более высокого порядка. Соответствующие вычисления ведут к появлению дифракционных максимумов более высокого порядка, которые отклоняются под углами $\pm 6 \theta, \pm 8 \theta, \pm 10 \theta \ldots$ и имеют задержки $\left|3 t_{R}\right|,\left|4 t_{R}\right|,\left|5 t_{R}\right| \ldots$. Также возникают дополнительные искажения для более низких дифракционных порядков. Поскольку в соответствии с (19) амплитуда сигнала $n$-го порядка дифракции пропорциональна величине $1 / n !$, то высокие порядки являются слабыми по интенсивности.

Естественно, возникает вопрос, каково распределение интенсивности считывающего импульса между разными порядками дифракции. Для точного анализа в суммах (19) и (20) необходимо учитывать все члены более высокого порядка. Приближенные оценки показывают, что каждый порядок имеет максимальную амплитуду при определенной экспозиции. Например, луч первого порядка дифракции имеет максимальную амплитуду при экспозиции $l|E|^{2} \approx 1 / x\left(|E|^{2}-\right.$ энергия записываемого импульса). При этом его интенсивность может составлять более чем $80 \%$ интенсивности распространяющегося прямо импульса, а импульс второго порядка дифракции, в свою очередь, в четыре раза слабее по сравнению с импульсом первого порядка. В таких условиях остальные порядки значительно слабее (третий, например, всего около $3 \%$ интенсивности первого и т. д.).

\section{Заключение}

Итак, при больших дозах облучения учет нелинейности процесса записи пространственно-временной голограммы приводит к появлению в восстановленном поле дополнительных дифракционных максимумов более высокого порядка, задержанных соответственно 2, 3, 4, ...-кратно по сравнению с линейной частью сигнала. При этом поправки применяются и к линейным членам, в результате чего их относительная интенсивность уменьшается. Кроме того, возникают разнообразные искажения восстановленного сигнала во всех порядках дифракции. Оценки показывают, что вклад высших порядков может достичь $10-20 \%$ в условиях, где эффективность рассеяния голограммы близка к максимальной.

Авторы выражают благодарность К. К. Ребане за поддержку и постоянный интерес к работе, П. Саари и А. Ребане за полезные обсуждения и советы, стимулирующие проведение данной работы. 
1. Ребане А. К., Каарли Р. К., Саари П. М. Письма в ЖЭТФ, 38, вып. 7, 320-323 (1983).

2. Rebane, A., Kaarli, R., Saari, P., Anijalg, A., Timpmann, K. Opt. Common., 47, № 3, 173-176 (1983).

3. Ребане А. К., Каарли Р. К., Саари П. М. Изв. АН ЭССР. Физ. Матем., 34, № 4, $444-448$ (1985).

4. Саари П. М., Ребане А. К. Изв. АН ЭССР. Физ. Матем., 33, № 3, 332-333 (1984).

5. Ребане $A . K$. Когерентный отклик и пространственно-временная голография на примесных системах с фотовыжиганием провалов. Автореф. дис. Тарту, 1984.

6. Гороховский А. А., Каарли Р. К., Ребане Л. А. Письма в ЖЭТФ, 20, вып. 7, $474-479$ (1974).

7. Кикас Я. В. Некоторые вопросы исследования электронно-колебательных спектров примесных молекул в неоднородных твердотельных матрицах. Автореф. канд. дис. Тарту, 1978.

8. Rebane, A. Gorohhovski, A., Kikas, J. Appl. Phys., B 29, 235-250 (1982).

9. Ребане K. К. Элементарная теория колебательной структуры примесных центров кристаллов. М., «Наука», 1968.

Институт физики

Академии наук Әстонской ССР
Поступила в редакцию 22/XII 1986

R. SARAPUU, R. KAARLI

\section{AEGRUUMILISTE HOLOGRAMMIDE MITTELINEAARNE SALVESTAMINE . SPEKTRAALSELT KÖRGSLEKTIIVSETES FOTOKROOMSETES KESKKONDADES}

Lähtudes varasematest eksperimentaalsetest ja teoreetilistest töödest, on esitatud aegruumilise hologrammi kirjutamise ja taastamise protsesside täpsustatud teoreetiline käsitlus. Vabadest ostsillaatoritest koosneva keskkonna mudeli alusel on esitatud ja vastuvõetavas lähenduses analüütiliselt ka lahendatud integraalvõrrandite süsteem valgusimpulsside levimise kirjeldamiseks kõrgselektiivsetes fotokroomsetes keskkondades. On näidatud, et aegruumilise hologrammi salvestusprotsessis kiirgusdoosi järgi teist järku mittelineaarsete liikmete arvestamine viib lugemisel vastavat järku difraktsioonimaksimumide tekkele, mis moodustuvad kahekordse viivisega, vōrreldes lineaarsete liikmetega. Uhtlasi on tehtud mittelineaarsusliikmete osatähtsuse ja struktuuri detailne analüüs.

\section{R. SARAPUU, R. KAARLI}

\section{NONLINEARITY OF THE RECORDING PROCESS OF TIME AND SPACE DOMAIN HOLOGRAMS IN HIGHLY SELECTIVE PHOTOCHROMIC MEDIA}

In this paper, the contribution of the nonlinearity of the recording process in time and space domain holography is investigated. To describe the propagation of short light pulses in a photochemically active medium integral, equations are derived and solved analytically in a proper approximation. It is shown that if the second-order terms describing the recording process are taken into account, the second-order diffraction occurs during reconstruction while its delay is twice longer than that for the replicas scattered to the first order. A detailed analysis of the dependence of the restoration process on the contribution of the nonlinearity of the recording process is made. 